\title{
Three-Dimensional Relationships between Hippocampal Synapses and Astrocytes
}

\author{
Rachel Ventura ${ }^{1}$ and Kristen M. Harris ${ }^{2}$ \\ ${ }^{1}$ Harvard College and 2 Program in Neuroscience, Harvard Medical School, Division of Neuroscience in the Department of \\ Neurology, Children's Hospital, Boston, Massachusetts 02115
}

Recent studies show that glutamate transporter-mediated currents occur in astrocytes when glutamate is released from hippocampal synapses. These transporters remove excess glutamate from the extracellular space, thereby facilitating synaptic input specificity and preventing neurotoxicity. Little is known about the position of astrocytic processes at hippocampal synapses. Serial electron microscopy and three-dimensional analyses were used to investigate structural relationships between astrocytes and synapses in stratum radiatum of hippocampal area CA1 in the mature rat in vivo and in slices. Only $57 \pm 11 \%$ of the synapses had astrocytic processes apposed to them. Of these, the astrocytic processes surrounded less than half $(0.43 \pm 22)$ of the synaptic interface. Other studies suggest that astrocytes extend processes toward higher concentrations of glutamate; thus the presence of astrocytic processes at particular hippocampal synapses might signal which ones are releasing glutamate. The distance between nearest neighboring synapses was usually $(\sim 95 \%)<1 \mu \mathrm{m}$. Astrocytic processes occurred along the extracellular path between 33\% of the neighboring synapses, neuronal processes occurred along the path between another $66 \%$ of the neighboring synapses, and only $1 \%$ of the synapses were close enough such that neither astrocytic nor neuronal processes occurred between them. These morphological arrangements suggest that the glutamate released at approximately two-thirds of hippocampal synapses might diffuse to other synapses, unless neuronal glutamate transporters are more effective than previously reported. The findings also suggest that physiological recordings made from hippocampal astrocytes do not uniformly sample the glutamate released from all hippocampal synapses.

Key words: astrocytes; serial electron microscopy; glutamate spillover; transporters; long-term potentiation; multiple synapse boutons
The location and distribution of astrocytic processes is important for regulating the extracellular milieu in the CNS. Astrocytes provide energy for neuronal function and modulate the formation and efficacy of synapses (Pfrieger and Barres, 1996, 1997; Laming et al., 1998; Smith, 1998). They regulate extracellular glutamate, via glutamate transporters (Rothstein et al., 1994; Chaudhry et al., 1995), and recycle glutamate via glutamine and intermediates of the tricarboxylic acid cycle (Schousboe et al., 1997; Sonnewald et al., 1997). By clearing excess glutamate from the extracellular space (ECS), astrocytes protect against excitotoxic glutamate concentrations that can lead to neuronal cell death (Choi, 1988; Rosenberg and Aizenman, 1989; Rosenberg et al., 1992; Mennerick et al., 1996; Rothstein et al., 1996; Porter and McCarthy, 1997). Astrocytes are also likely to communicate directly with neurons via cell-cell adhesion junctions (Spacek and Harris, 1998) and intercellular calcium signaling (Parpura et al., 1994; Porter and McCarthy, 1996; Verkhratsky and Kettenmann, 1996; Vernadakis, 1996).

Glia occupy $\sim 50 \%$ of the total brain volume (Peters et al.,

\footnotetext{
Received Dec. 17, 1998; revised May 26, 1999; accepted May 27, 1999.

This work was supported by National Institutes of Health Grants NS21184 and MH/DA57351, with the latter funded jointly by the National Institute of Mental Health, National Institute on Drug Abuse, and National Aeronautics and Space Administration (K.M.H.), and the Mental Retardation Research Center Grant P30-HD18655 (Dr. Joseph Volpe, PI). We thank Dr. John Fiala for creation of the IGLtrace reconstruction system. We thank Dr. Craig Jahr, Dr. Paul Rosenberg, and Dr. John Fiala for helpful discussions about this work.

Correspondence should be addressed to Dr. Kristen M. Harris, Department of Neurology, Children's Hospital, Enders 208, 300 Longwood Avenue, Boston, MA 02115 .

Copyright (C) 1999 Society for Neuroscience $\quad 0270-6474 / 99 / 196897-10 \$ 05.00 / 0$
}

1991; Laming et al., 1998). However, glial processes are not uniformly distributed in different brain regions. In the cerebellar cortex, nearly all of the parallel and climbing fiber synapses are completely ensheathed by processes of the Bergmann glia (Spacek, 1985). Physiological recordings show that these glia are responsive to glutamate released at the synapses (Bergles et al., 1997; Linden, 1997) and that the glial transporter-mediated currents potentiate in parallel with long-term potentiation (LTP) at the synapses (Linden, 1997, 1998). In contrast, only 29\% of neocortical synapses are contacted by astrocytes, and these are not fully surrounded by the astrocytic processes (Spacek, 1985). Furthermore, the structural relationships between astrocytes and synapses can change during development, in response to exogeneously applied glutamate, and with altered neuronal function (Pomeroy and Purves, 1988; Cornell-Bell et al., 1990; Sirevaag and Greenough, 1991; Harris and Rosenberg, 1993; Hawrylak et al., 1993; Anderson et al., 1994; Jones and Greenough, 1996; Theodosis and MacVicar, 1996).

Recent whole-cell recordings from astrocytes show glutamate transporter currents in response to glutamate released at hippocampal CA1 synapses (Bergles and Jahr, 1997). These transporter currents are potentiated for a few minutes during posttetanic potentiation, but not during LTP of the hippocampal synapses (Diamond et al., 1998; Luscher et al., 1998), in contrast with the cerebellar Bergmann glia. Other studies in hippocampus suggest that glutamate released at one synapse might diff use to neighboring synapses (Harris, 1995; Kullmann et al., 1996; Barbour and Hausser, 1997; Engert and Bonhoeffer, 1997; Malenka and Nicoll, 1997; Kullmann and Asztely, 1998). This "glutamate 
spillover" could facilitate synchronization of synaptic inputs but also could reduce synaptic input specificity.

Here serial electron microscopy (EM) and three-dimensional (3D) analyses were used to determine the structural features of hippocampal synapses and neighboring astrocytic processes that might regulate glutamate. In addition, the distance of the extracellular path between neighboring synapses was measured, and the structural components along the path that might affect the diffusion of glutamate were delineated.

\section{MATERIALS AND METHODS}

All of our protocols undergo yearly review by the Animal Care and Use Committee at Children's Hospital according to the National Institutes of Health guidelines. EM series from previous studies were used. Some were from the hippocampi of two male rats (rat 1, $137 \mathrm{gm}, 39 \mathrm{~d}$; rat 2, 310 gm, $77 \mathrm{~d}$ ), which were prepared under deep pentobarbital anesthesia by intravascular perfusion with $2.5 \%$ glutaraldehyde, $2 \%$ paraformalde-

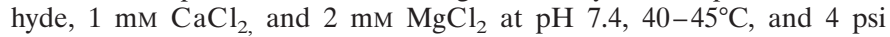
pressure (Harris and Stevens, 1989; Harris et al., 1992). Other series were from hippocampal slices from two male rats (rat 3, $326 \mathrm{gm}, 53 \mathrm{~d}$; rat 4, $279 \mathrm{gm}, 60 \mathrm{~d})$, which had been prepared by microwave-enhanced fixation (Sorra and Harris, 1998). All of the series were located 150-250 $\mu \mathrm{m}$ from the hippocampal CA1 pyramidal cell body layer in the middle of stratum radiatum.

Table 1 summarizes the sources of each sample used for the analyses described in Results. The first two samples of rat 1 were used in all of the analyses. The other samples were used to assess the generality of the results in different animals, as well as in hippocampal slices maintained in vitro.

New 3D reconstructions were completed using software programs developed in the Image Graphics Laboratory at Children's Hospital [available through http://synapses.tch.harvard.edu (until October, 1999) and http://www.nimh.nih.gov/neuroinformatics/]. Photographs from serial sections were scanned using the HP Scanjet $4 \mathrm{C}$ scanner, and then the images were digitally rotated and adjusted in the $x-y$ plane to obtain optimal alignment. Traces were superimposed on objects of interest and volumetric, areal, or linear dimensions were computed via calibrated pixels as determined by a calibration grid (Ted Pella, Inc., Redding, CA) that was originally photographed and then scanned with each series. 3D surfaces were rendered using 3D Studio Max (Kinetix, San Francisco, CA).

Statistics were performed using SigmaStat (Jandel Scientific), and all data are represented either as the individual sample points distributed around the mean or as the mean $\pm \mathrm{SD}$, depending on the specific analysis described in Results.

\section{RESULTS}

\section{Astrocytic content in stratum radiatum of hippocampal area CA1}

Astrocytic processes were identified by their irregular, stellate shape and by the presence of glycogen granules and bundles of intermediate filaments (data not shown) in a relatively clear cytoplasm (Fig. 1a) (also see Peters et al., 1991). Astrocytic content in the neuropil of stratum radiatum was estimated from 23 randomly selected sections obtained from each of the four rats (analysis 1, Table 1). The astrocytic content was determined by outlining and computing the area of all astrocytic profiles and dividing by the total area on each section. Fifteen of these sections had only astrocytic processes, which occupied $4 \pm 1 \%$ of the total area. Eight samples also had a portion of an astrocytic cell body, which together with the astrocytic processes occupied $7 \pm 2 \%$ of the total area.

\section{D reconstructions of synaptic complexes including astrocytic profiles}

Twenty-three representative synapses from rats 1 and 2 were selected for complete 3D reconstruction of the synaptic complexes including the presynaptic bouton, postsynaptic spine, and associated astrocytic processes (Fig. $1 b-d$, Table 1). The synaptic complexes had postsynaptic densities (PSDs) with continuous "macular" (Fig. 1b) shapes or with regions that were "perforated" by electron lucent areas (Fig. 1c). Both single synapse boutons (SSBs) (Figs. 1b,c) and multiple synapse boutons (MSBs) (Fig. $1 d)$ were present. The synaptic complexes occupied from 0.5 to $1.2 \mu \mathrm{m}^{3}$.

\section{The axon-spine interface}

The axon-spine interfaces have both synaptic and nonsynaptic components. The synaptic interface has a widened synaptic cleft bordered by vesicles in the presynaptic axonal bouton and a PSD in the dendritic spine (Fig. 2a). The nonsynaptic interface has a thin extracellular space bordered by spine and bouton membranes without specialization (Fig. $2 a$ ). The nonsynaptic interface may also contain molecules important for synaptic function, such as those involved in endocytosis or glutamate transport. The perimeter of the axon-spine interface is where substances secreted into the synaptic cleft might escape and diff use to neighboring synapses.

The total area of the axon-spine interface was measured by $3 \mathrm{D}$ reconstruction for 187 synapses from rats 1 and 2 (Table 1). The synaptic interface was determined by measuring the area of the PSD. The difference between the total area of the axon-spine interface and the area of the PSD equaled the nonsynaptic interface area. The areas of the synaptic and nonsynaptic interfaces scaled proportionately (Fig. $2 b, c)(r=0.66)$. The axonspine interface of the macular synapses ranged from 0.02 to 0.23

\begin{tabular}{|c|c|c|c|c|c|}
\hline Rat no. & $\begin{array}{l}\text { Sample } \\
\text { identification }\end{array}$ & $\begin{array}{l}\text { Number of } \\
\text { synapses }\end{array}$ & $\begin{array}{l}\text { Number of } \\
\text { sections }\end{array}$ & Volume $\left(\mu \mathrm{m}^{3}\right)$ & Analyses \\
\hline \multirow[t]{4}{*}{1} & $\mathrm{k} 21$ & 82 & 56 & 74 & $1,2,3,4,5$ \\
\hline & k34_dn10 & 66 & 33 & 42 & $1,2,3,4,5$ \\
\hline & k34_dn21 & 21 & 31 & 21 & $2,3,4,5$ \\
\hline & $\mathrm{k} 24$ & 9 & $29-40$ & & 2 \\
\hline 2 & $\mathrm{k} 18$ & 9 & 71 & & $1,2,3$ \\
\hline \multirow[t]{2}{*}{3} & ks69_ODSC & 60 & 34 & $\begin{array}{c}130 \\
\text { Area }\left(\mu \mathrm{m}^{2}\right) \\
\end{array}$ & $1, \quad 4$ \\
\hline & ks69_XTYZ & & 2 & 276 & 1 \\
\hline \multirow[t]{2}{*}{4} & ks68_TTSM & & 2 & 260 & 1 \\
\hline & ks68_YNLP & & 2 & 376 & 1 \\
\hline
\end{tabular}

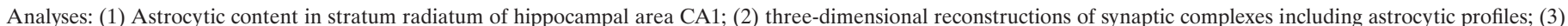

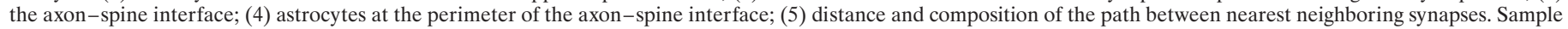
volumes were estimated by measuring the section area and then multiplying by section thickness and the number of sections. 
a

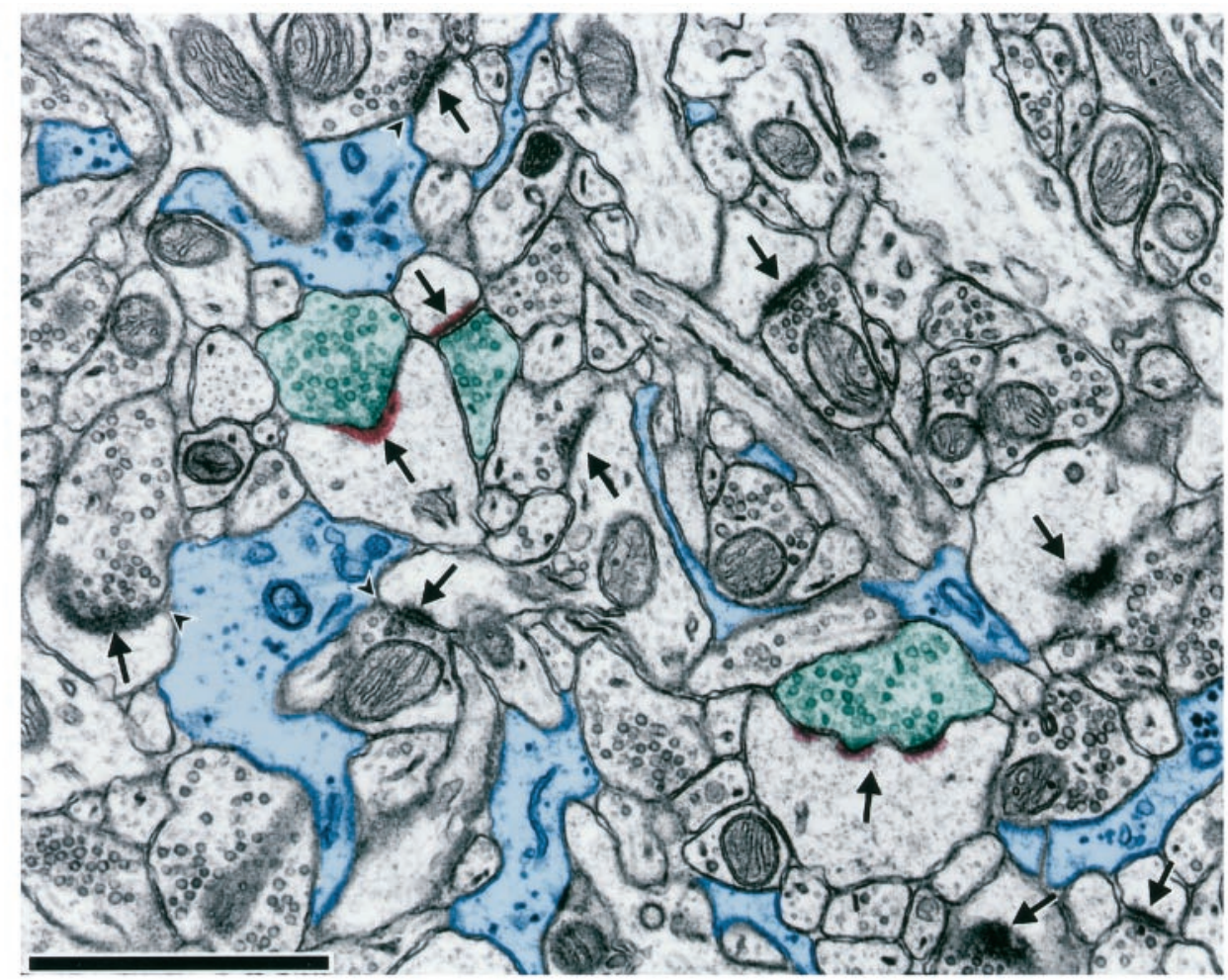

b
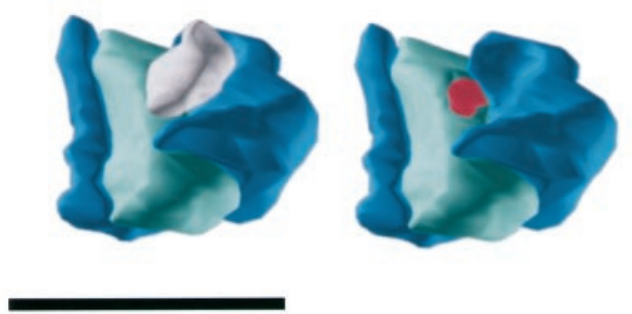

C
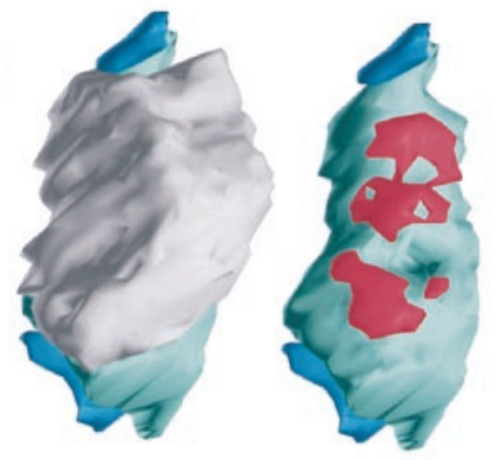

d
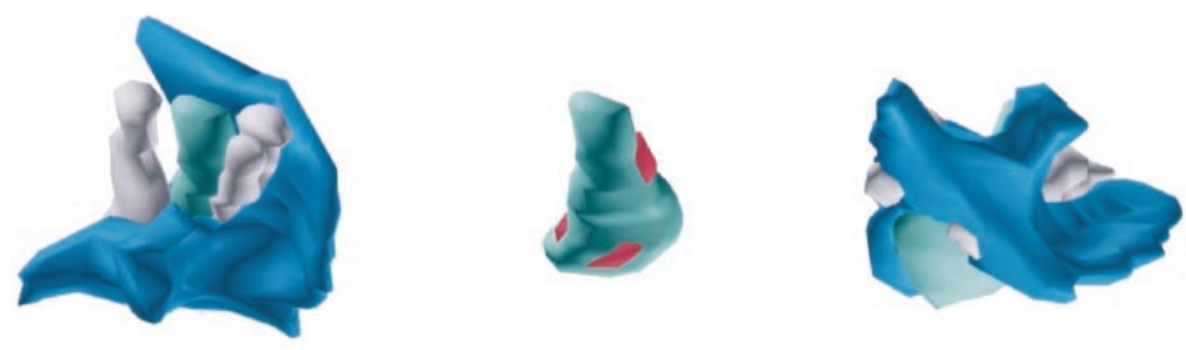

Figure 1. Astrocytic profiles and 3D reconstructions illustrating their relationships to synapses in the middle of stratum radiatum of hippocampal area CA1. $a$, Astrocytic profiles are illustrated (blue) on a single thin section in the vicinity of 11 synapses (arrows). The identity of each of the astrocytic profiles occurring on this single EM section was confirmed by viewing serial sections. On this one section, three synapses have astrocytic profiles at their perimeters (arrowheads). To identify whether astrocytic processes occurred at the perimeter of the other synapses, they were viewed through serial sections, and four more of the synapses were found to have astrocytic profiles at their perimeters, for a total of seven. $b-d$, 3D reconstructions illustrate astrocytic profiles (blue), boutons (green), spines ( gray), and PSDs (red). Astrocytic profiles surround (b) $50 \%$ of the perimeter of this macular synapse and $(c) 3 \%$ of the perimeter of a perforated synapse, both occurring on SSBs. In $d$, three synapses occur with a single presynaptic bouton, called a multiple synapse bouton, and a single astrocytic process surrounds 75,64 , or $100 \%$ of the perimeter of each synapse from left to right, respectively. Scale bars: $1 \mu \mathrm{m}$ (shown in $b$ for $b-d$ ).

$\mu \mathrm{m}^{2}$ and had $48 \pm 1 \%$ nonsynaptic interface. In contrast, the axon-spine interface of the perforated synapses ranged from 0.25 to $1.1 \mu \mathrm{m}^{2}$ and had $63 \pm 2 \%$ nonsynaptic interface (MannWhitney rank sum test, $t=2167 ; p<0.001$ ). The volumes of the synaptic clefts were estimated by multiplying the interface area times cleft width for representative small and large synapses. The volume of the synaptic cleft ranged from $0.15 \times 10^{-3}$ to $2.1 \times$ $10^{-3} \mu \mathrm{m}^{3}$ at macular synapses, and $1.7 \times 10^{-3}$ to $7.6 \times 10^{-3}$ $\mu \mathrm{m}^{3}$ at the perforated synapses. The volume of the nonsynaptic interface ranged from $0.005 \times 10^{-3}$ to $3.5 \times 10^{-3} \mu \mathrm{m}^{3}$ at the macular synapses and $2.1 \times 10^{-3}$ to $17 \times 10^{-3} \mu \mathrm{m}^{3}$ at the perforated synapses.

\section{Astrocytes at the perimeter of the axon-spine interface}

Astrocytes surrounding the perimeter of the axon-spine interface are ideally situated to regulate glutamate and other substances released at synapses. To determine what percentage of hippocampal synapses had astrocytes at their perimeter, all complete synaptic complexes within the series from rats 1 and 3 were 
b
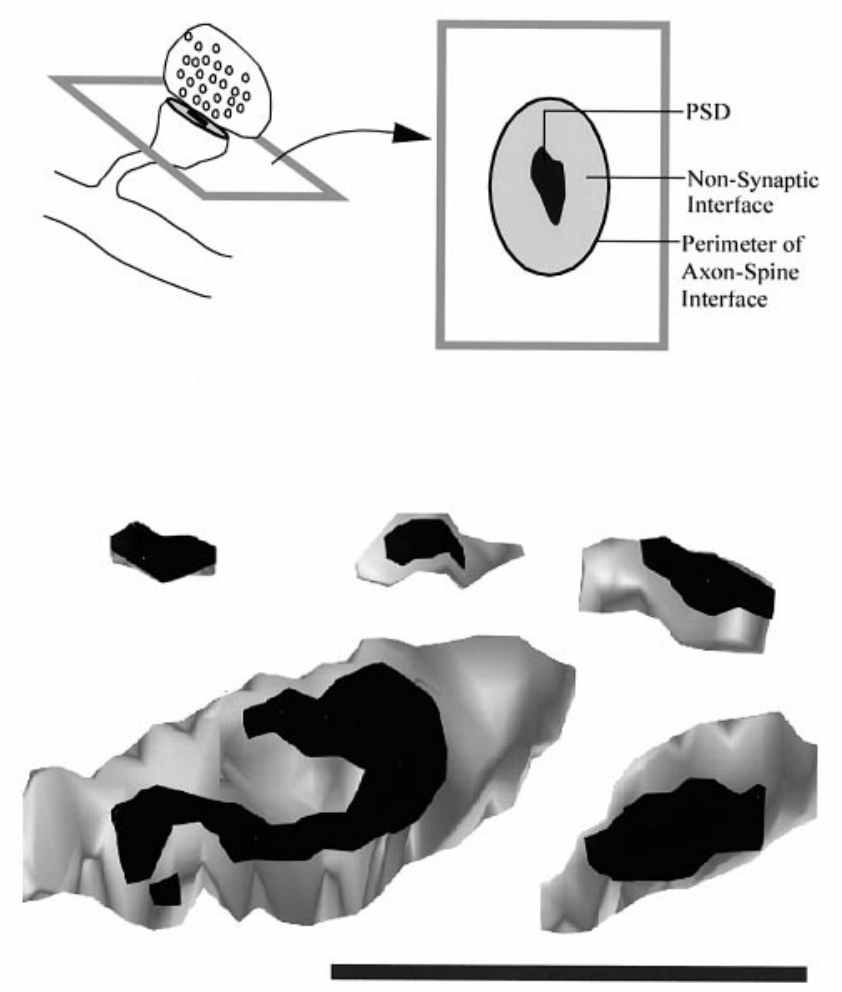

Figure 2. The axon-spine interface. $a$, Schematic illustration of the PSD, the nonsynaptic interface, and the perimeter of the axon-spine interface viewed en face. $b$, Five 3D reconstructions of the axon-spine interface arranged clockwise in order of increasing PSD size. These reconstructions demonstrate the variability in the sizes and shape of the PSDs and the nonsynaptic interfaces. Scale bar: $1 \mu \mathrm{m}$. $c$, Larger synapses have larger nonsynaptic interfaces as well.

C

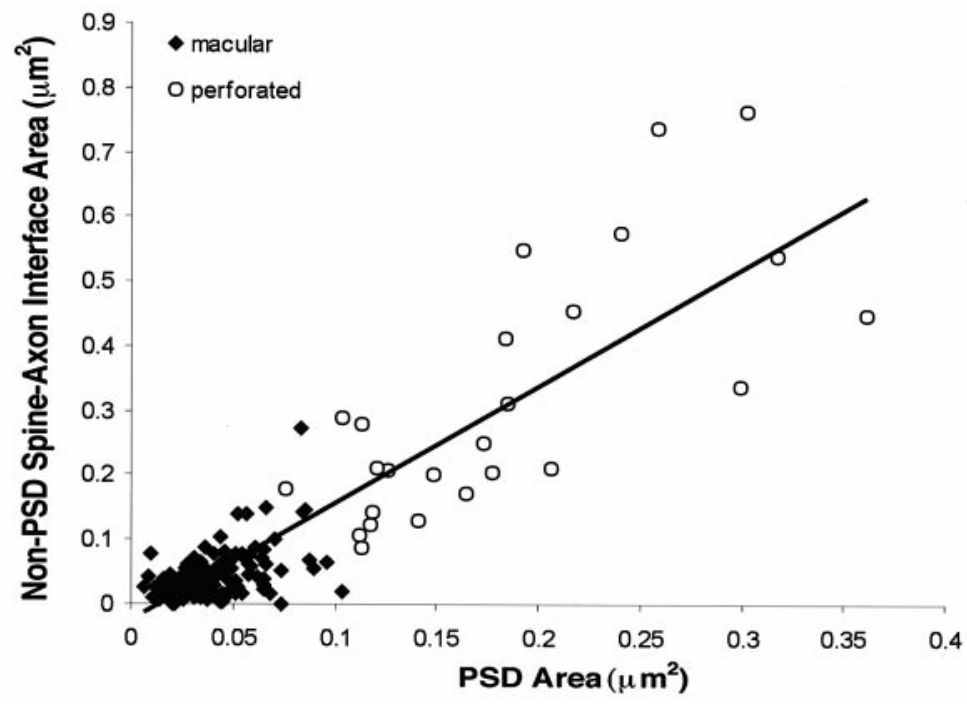

analyzed (Fig. 3, Table 1). Because the sample volumes were substantially larger $\left(21-130 \mu^{3}\right)$ (Table 1) than the largest synaptic complex $\left(1.2 \mu \mathrm{m}^{3}\right.$, see above), these volumetric analyses contained representative sizes and types of synapses. There were 229 complete synaptic complexes in these sample volumes. Of these, 197 had macular and 32 had perforated PSDs, and 187 occurred on SSBs and 42 occurred on MSBs. Astrocytic profiles occurred at the perimeter of the axon-spine interface of $57 \%$ of the synapses (Fig. 3); 44\% were astrocytic processes, and 13\% were astrocytic cell bodies. Astrocytes occurred at $52 \%$ of the macular synapses, at $88 \%$ of the perforated synapses, at $61 \%$ of the SSBs, and at $40 \%$ of the MSBs (Fig. 3).

The 3D reconstructions illustrate a high variation in how much of the perimeter of the axon-spine interface was surrounded by astrocytic profiles (Fig. 1). The following procedure was used to estimate the fraction of this perimeter that was surrounded for the 131 synapses with astrocytic profiles. On each section of a cross-sectioned synapse, the perimeter had two parts, one at each edge of the axon-spine interface. When the cap (i.e., the last section) of the axon-spine interface was reached, the next section 


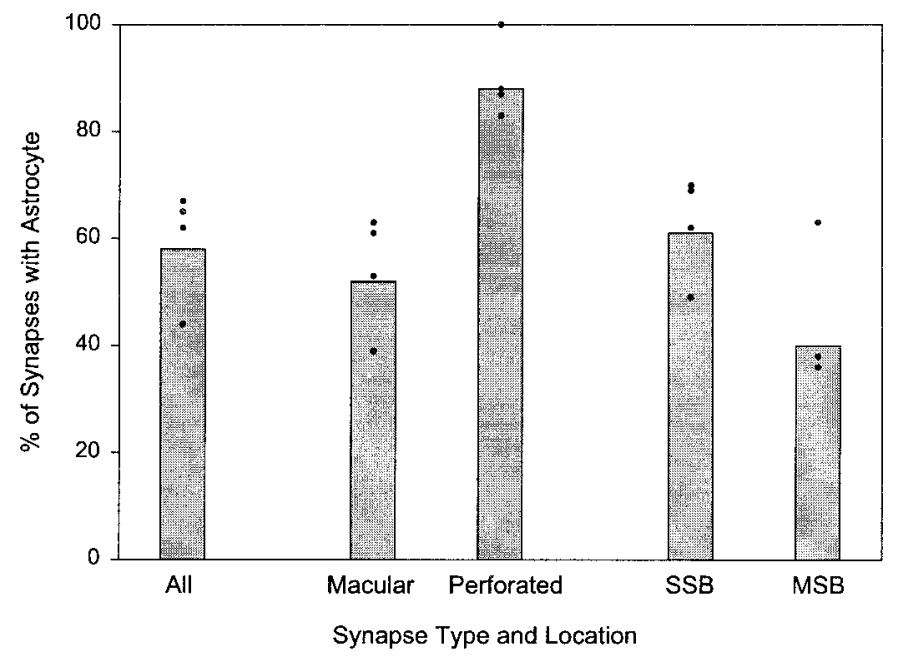

Figure 3. Percentage of synapses with astrocytic profiles at the perimeter of the axon-spine interface. (Height of bar $=$ the mean across 4 series volumes; individual values are superimposed; total $n=229$ synapses.)

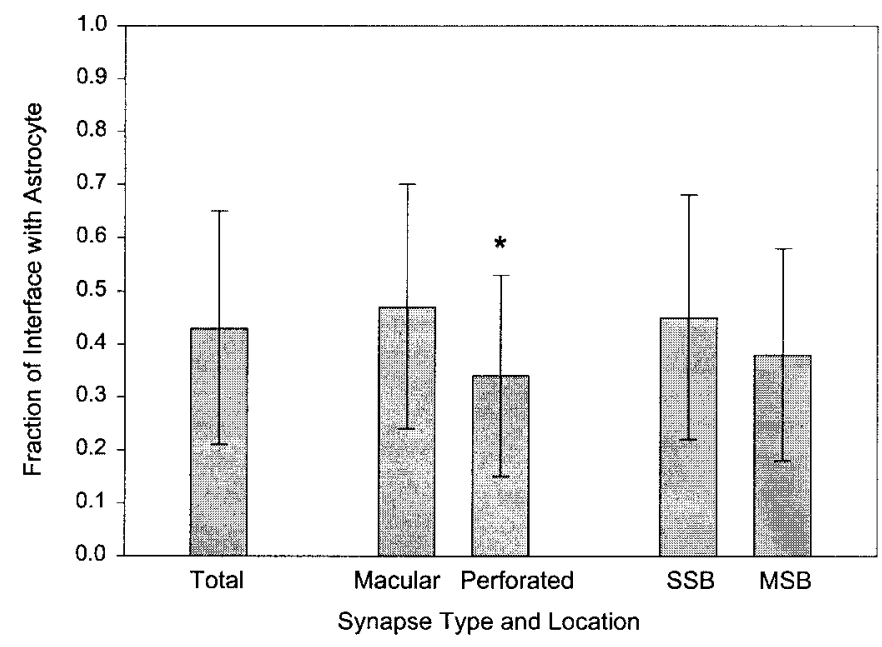

Figure 4. Fraction of the perimeter of individual hippocampal synapses that is surrounded by astrocytic profiles (mean $\pm \mathrm{SD} ; n=131$ synapses). The amount of astrocyte surrounding the perimeter at macular synapses $(n=103)$ was greater than at perforated synapses $\left(n=28 ;{ }^{*} p<0.01\right)$. None of the other differences reached statistical significance.

was evaluated to determine whether astrocytic processes surrounded the cap. Then the fraction of edges with an astrocytic profile was determined. The fraction of this perimeter that was surrounded by astrocytic profiles was $0.43 \pm 0.22$ (Fig. 4). At macular synapses the fraction that was surrounded was $0.47 \pm$ 0.23 compared with $0.34 \pm 0.19$ at perforated synapses $(t=2.678$; $p<0.01)$. The degree to which the astrocytic profiles surrounded synapses on SSBs $(0.45 \pm 0.23, n=114)$ versus MSBs $(0.38 \pm$ $0.20, n=17)$ was not significantly different $(t=1.121 ; p=0.264)$. There tended to be more astrocytic coverage at synapses with a smaller nonsynaptic interface, although this trend did not reach statistical significance $(r=-0.12, p=0.09)$.

\section{Distance and composition of the path between nearest} neighboring synapses

If glutamate or other substances escape from the synaptic cleft, their impact on neighboring synapses would be affected by the distance they must diffuse. In addition the occurrence of astro- cytic or neuronal processes along the path might impede diff usion structurally or via binding and subsequent transport. The nearest neighboring (NN) synapse could be identified for 141 of the synapses from rat 1 (Table 1). The NN synapse was found either on the same section $(n=98)$, as is shown in Figure 5, or at an angle through adjacent serial sections $(n=43)$. The length of the tortuous path through extracellular space was measured between neighboring synapses. When the path traversed more than one section, the Pythagorean theorem $\left[c=\operatorname{sqrt}\left(a^{2}+b^{2}\right)\right]$ was applied. The length across adjacent sections $(c)$ was computed by measuring the linear displacement $(a)$ and then counting the number of sections traversed and multiplying by section thickness $(b)$.

The path between NN synapses often had neuronal (Fig. 5a,b) and/or astrocytic profiles between them (Fig. $5 c, d$ ). Only rarely were the synapses so close to one another that neither neuronal nor astrocytic profiles separated them (Fig. $5 e, f$ ). The NN synapse occurred either on the same bouton (Fig. $5 b, N N 1 \leftrightarrow 2$ ) or on a different bouton (Fig. $5 b, N N 2 \leftrightarrow 3$ ). Although $22 \%$ of the synapses occurred on MSBs (Fig. 6a), most of them had NN synapses on a different bouton (Fig. $6 b$ ). Overall, $88 \%$ of the NN synapses occurred on different presynaptic boutons; thus substances escaping from their axon-spine interfaces could reduce input specificity.

Neurotransmitter can be released at the edge and the center of the synaptic interface; hence both edge-to-edge and center-tocenter distances are relevant for understanding diff usion between synapses. The shortest distances through extracellular space between the edges of the PSDs on NN synapses were measured. Then the additional distance from the center to the edge of the synapse was computed by measuring the total PSD area and calculating the average radius, as though the PSD were circular. These radii were added to the edge-to-edge distances to yield the approximate center-to-center distances between NN synapses. The distances between edges of NN synapses ranged from 0.063 to $1.4 \mu \mathrm{m}$, with a mean of $0.42 \pm 0.2 \mu \mathrm{m}$. The distances between the calculated centers of synapses ranged from 0.26 to $1.8 \mu \mathrm{m}$, with a mean of $0.65 \pm 0.3 \mu \mathrm{m}$ (Fig. $7 a$ ).

Approximately $33 \%$ of the NN synapse pairs had an astrocytic profile somewhere along the shortest path between them (Fig. $7 b)$. Another $65 \%$ had neuronal membranes and processes between them, whereas $<1.5 \%$ of the synapses had neither astrocytic nor neuronal membrane between them. Distances between nearest neighboring synapses were longer when astrocytes occurred along the path $(0.53 \pm 0.24 ; n=48)$ than when they did not $(0.36 \pm 0.19 ; n=93$; Mann-Whitney rank sum test, $t=$ $4481 ; p<0.001)$.

\section{DISCUSSION}

The focus of this work has been to delineate which variations in the structural features of hippocampal spine synapses and their associated astrocytic processes might influence the regulation of glutamate. The results show that there is large variation in the composition of the axon-spine interface, in whether astrocytic processes surround this interface, and in whether astrocytic or neuronal processes occur along the path between neighboring synapses. This variation in structure suggests that glutamate escapes nonuniformly from hippocampal synapses and that astrocytes regulate these synapses unequally.

Thin sheets of astrocytic processes intermingle with dendrites, axons, and synapses, and occupy only $\sim 5 \%$ of the neuropil in stratum radiatum of area CA1. This $5 \%$ value compares well with 


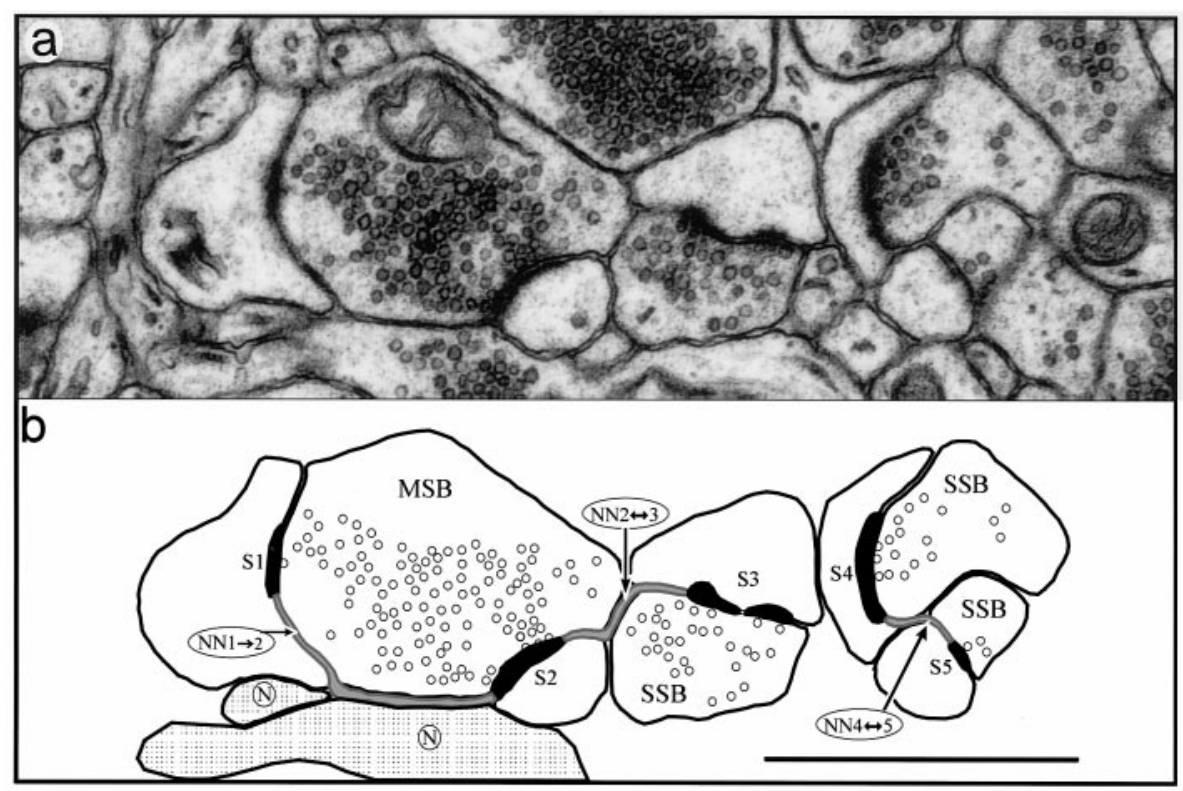

Figure 5. Five pairs of nearest neighboring $(N N)$ synapses. EM $(a)$ and schematic $(b)$ illustrations of the extracellular path among a cluster of neighboring synapses that have neuronal $(N$, dotted) processes or neuronal membranes of the axon-spine interface between them. NN path lengths ( gray) denoted by double arrows signify mutual NN paths, whereas those with single arrows signify one-way NN paths. Path lengths in $b$ from left to right are $0.85,0.47$, and $0.15 \mu \mathrm{m} . c, d$, EM and schematic representation of NN synapses that have both neuronal and astrocytic $(A$, striped $)$ profiles along that path between them, which is $1.1 \mu \mathrm{m}$. In $e$ and $f$, the two synapses are immediately adjacent to one another with almost no distance $(<0.01 \mu \mathrm{m})$ between them. In this case, the two NN synapses are on an MSB; in the only other case where synapses were this close to one another, the NN synapses were on two different boutons. Scale bar (shown in $b$ ): $a, b, 1 \mu \mathrm{m}$; (shown in $d$ ) $c-f, 1 \mu \mathrm{m}$.
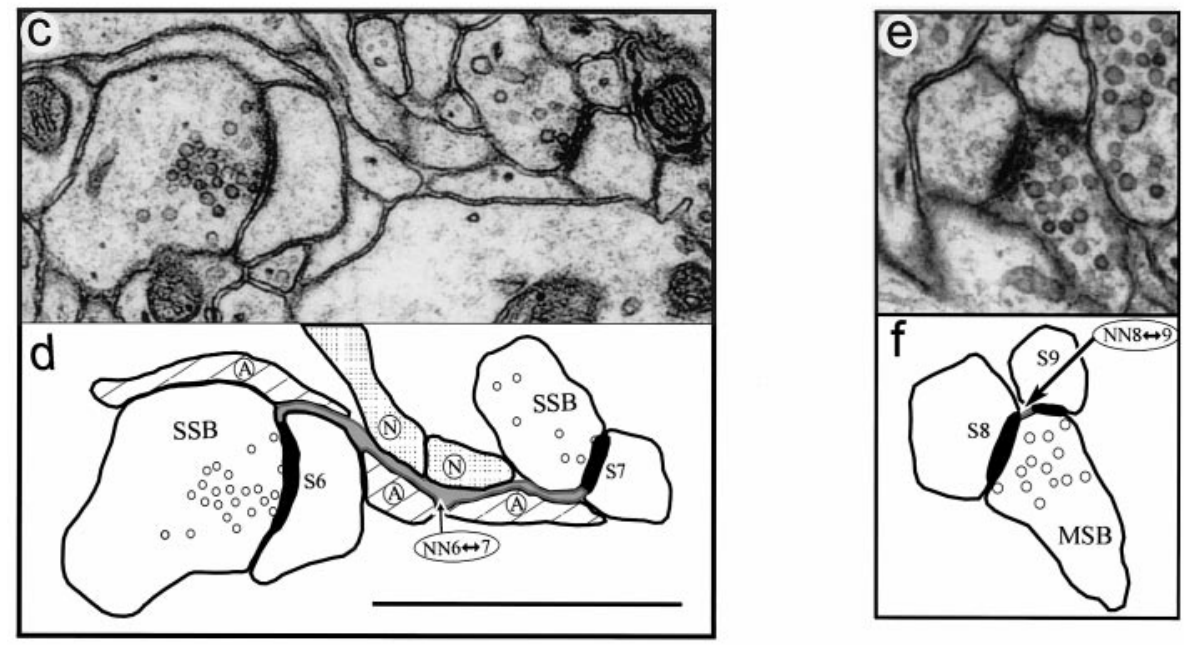

stereological measurements in area CA1 and neocortex (Hawrylak et al., 1993; Jones, Greenough, 1996) but contrasts with cerebellar cortex (Palay and Chan-Palay, 1974). Other studies show that $\sim 10 \%$ of all cell membranes in hippocampus are astrocytic, contrasting with $\sim 27 \%$ in cerebellar cortex (Lehre and Danbolt, 1998).

The disposition of astrocytic processes around synapses is also variable. A close apposition of astrocytic processes should facilitate binding and transport of synaptically released glutamate into astrocytes (Barbour et al., 1994; Rothstein et al., 1994; Chaudhry et al., 1995; Gundersen et al., 1995; Takahashi et al., 1997). In cerebellar cortex, 3D reconstructions show that glial processes often form a collar around the perimeter of the axon-spine interface (Palay and Chan-Palay, 1974; Spacek, 1985). Single EM sections from hippocampus show synapses with or without astrocytic processes (Lehre and Danbolt, 1998; Rusakov and Kullmann, 1998a,b). Our quantitative 3D analyses reveal that most of the large perforated synapses have astrocytes at their perimeters, although only approximately one-third of the perimeter of indi- vidual perforated synapses is surrounded. In contrast, fewer small macular synapses have astrocytes at their perimeter, but those that do are more completely surrounded.

This nonuniform distribution of astrocytic processes raises the question of whether they are randomly distributed or grow toward specific synapses. Astrocytes in cell culture extend processes toward substances that are released at synapses, including glutamate (Hatten, 1985; Cornell-Bell et al., 1990; Matsutani and Yamamoto, 1997). Several factors will determine whether sufficient glutamate or other astrotrophic substances escape from the synaptic cleft, including the amount released and then diluted in the axon-spine interface. Glutamate must escape from the synaptic cleft to the perimeter of at least some of the hippocampal synapses, because glutamate transporter currents occur in astrocytes when the synapses are activated (Bergles and Jahr, 1997). The probability of glutamate being released during synaptic transmission varies greatly among hippocampal synapses (Hessler et al., 1993; Murphy and Segal, 1997; Liu et al., 1999; Ma et al., 1999). Large perforated synapses have many docked vesicles and 

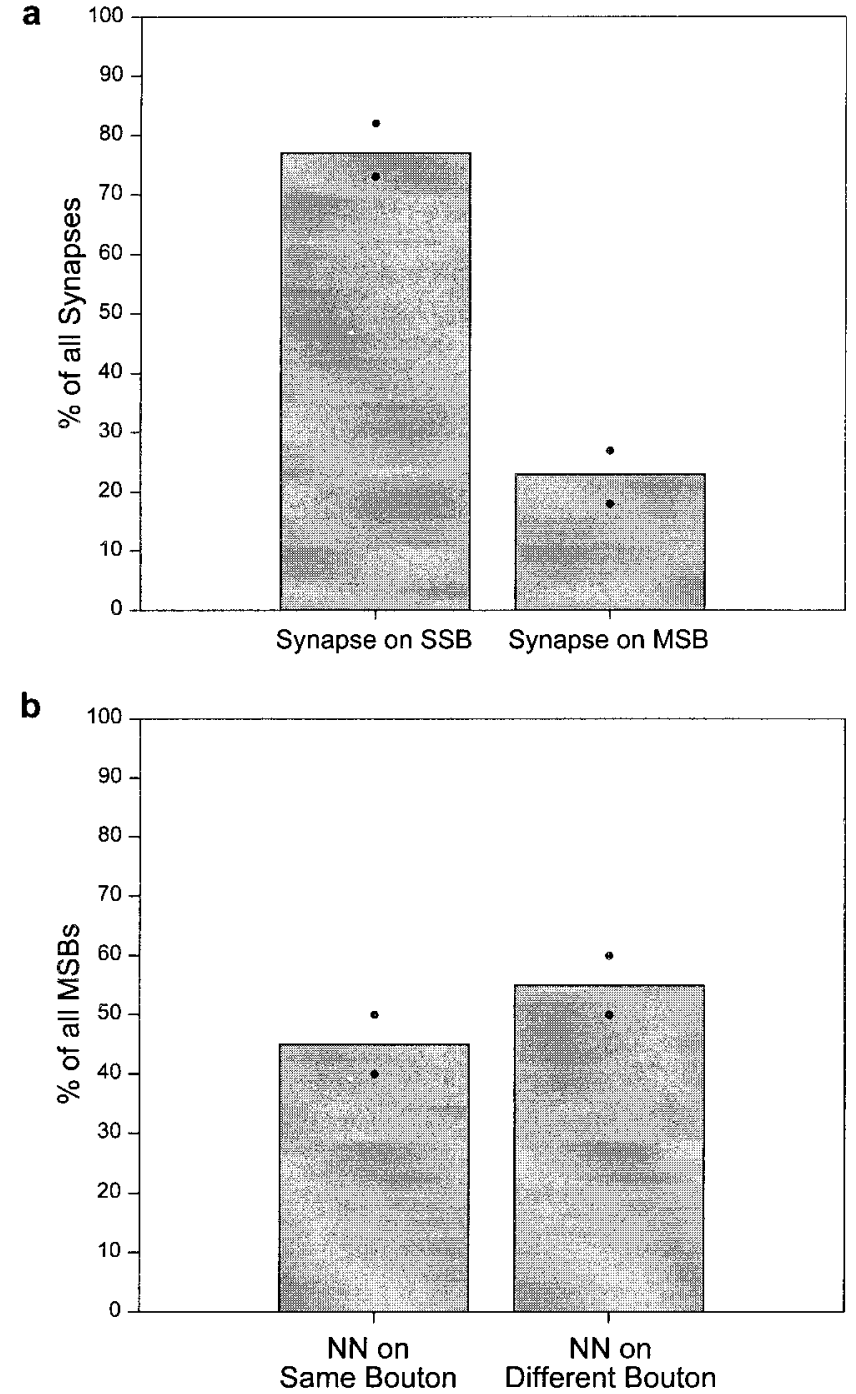

Figure 6. Percentage of synapses on SSBs and MSBs. $a$, Of the 229 synapses evaluated, 187 were on SSBs and 42 were on MSBs. $b$, Only 32 of the synapses on MSBs had the NNs within the series volume: 14 had their NN on the same MSB, whereas 18 had their NNs on a different bouton.

thus might have a high probability of release (Harris and Sultan, 1995), which could explain why almost $80 \%$ of them have some astrocytic processes at their perimeters. However, the large axon-spine interface might substantially dilute the glutamate, which might explain why only one-third of their perimeter is surrounded by astrocytic processes. The smaller macular synapses have fewer docked presynaptic vesicles and thus might have a lower probability of releasing glutamate (Harris and Sultan, 1995), which might explain why almost one-half of the smaller macular synapses do not have astrocytic processes at their perimeters. However, when glutamate is released from a small synapse, the small axon-spine interface will produce less dilution. Thus, glutamate released from a small synapse might escape from many parts of the perimeter (i.e., $47 \%$ on average). These observations are consistent with the hypothesis that astrocytic processes preferentially surround synapses that have more glutamate escaping from their perimeters.

Whether a particular synapse will sense the glutamate that a

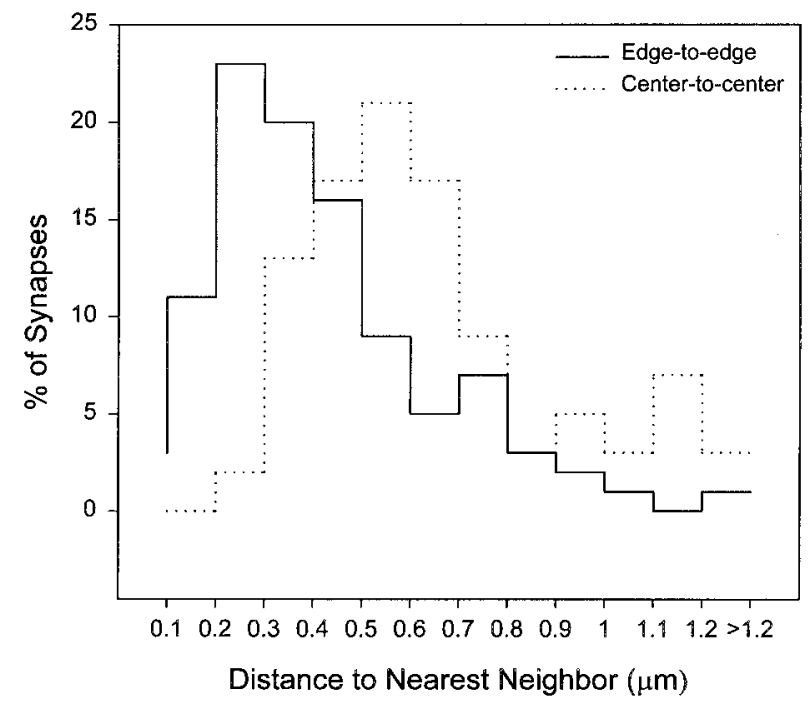

b

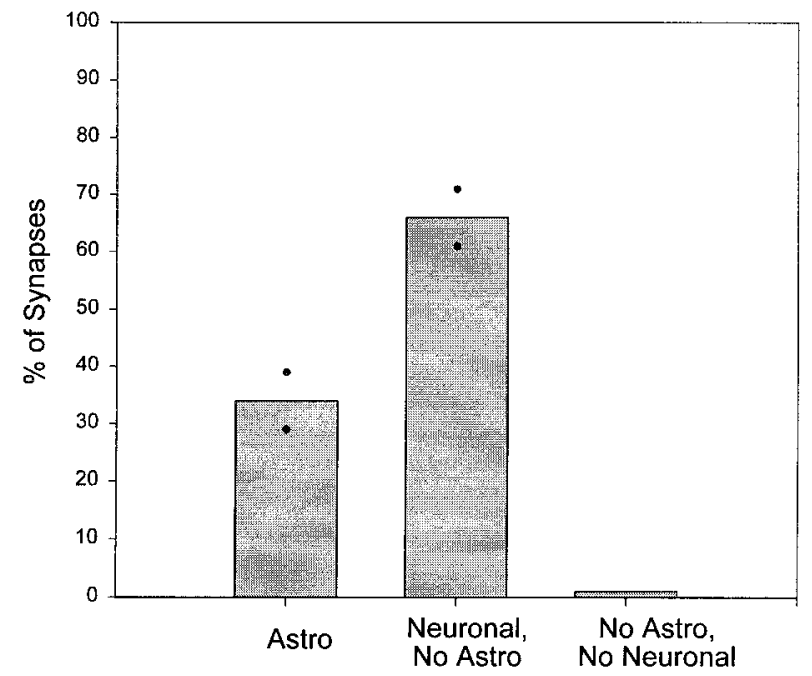

Figure 7. Distances and composition of processes along the paths between NN synapses. $a$, These measurements were obtained for 141 synapses. The mean edge-to-edge path length was $0.42 \pm 0.2 \mu \mathrm{m}$, and the mean center-to-center path length was $0.65 \pm 0.3 \mu \mathrm{m}$. $b$, Of these, the path between $48 \mathrm{NN}$ synapses had both astrocytic and neuronal membrane along it, whereas 91 had neuronal but no astrocytic membrane; only two pairs of $\mathrm{NN}$ synapses had neither astrocytic nor neuronal membrane between them.

escapes from the perimeter of its neighbor depends on uptake and dilution in the extracellular space between them. Because most of the neighboring synapses occur on different presynaptic boutons, glutamate diffusing between them will reduce input specificity. The specific effect will depend on the precise location of the glutamate receptors at the edge or center of the PSD because of their different affinities for glutamate (Lujan et al., 1997) How well EM images represent the volume of ECS is controversial. Others have estimated that ECS should occupy 20\% of living brain volume (Nicholson and Sykova, 1998). Some of the ECS might be lost during processing of fixed tissue because the remaining ECS volume appears to be $<20 \%$, and there is a net $5-15 \%$ overall shrinkage in fixed brain (Hillman and Deutsch, 1978; Cragg, 1980; Schuz and Palm, 1989). Others suggest that there is no net shrinkage or loss of ECS in the EM images, and 

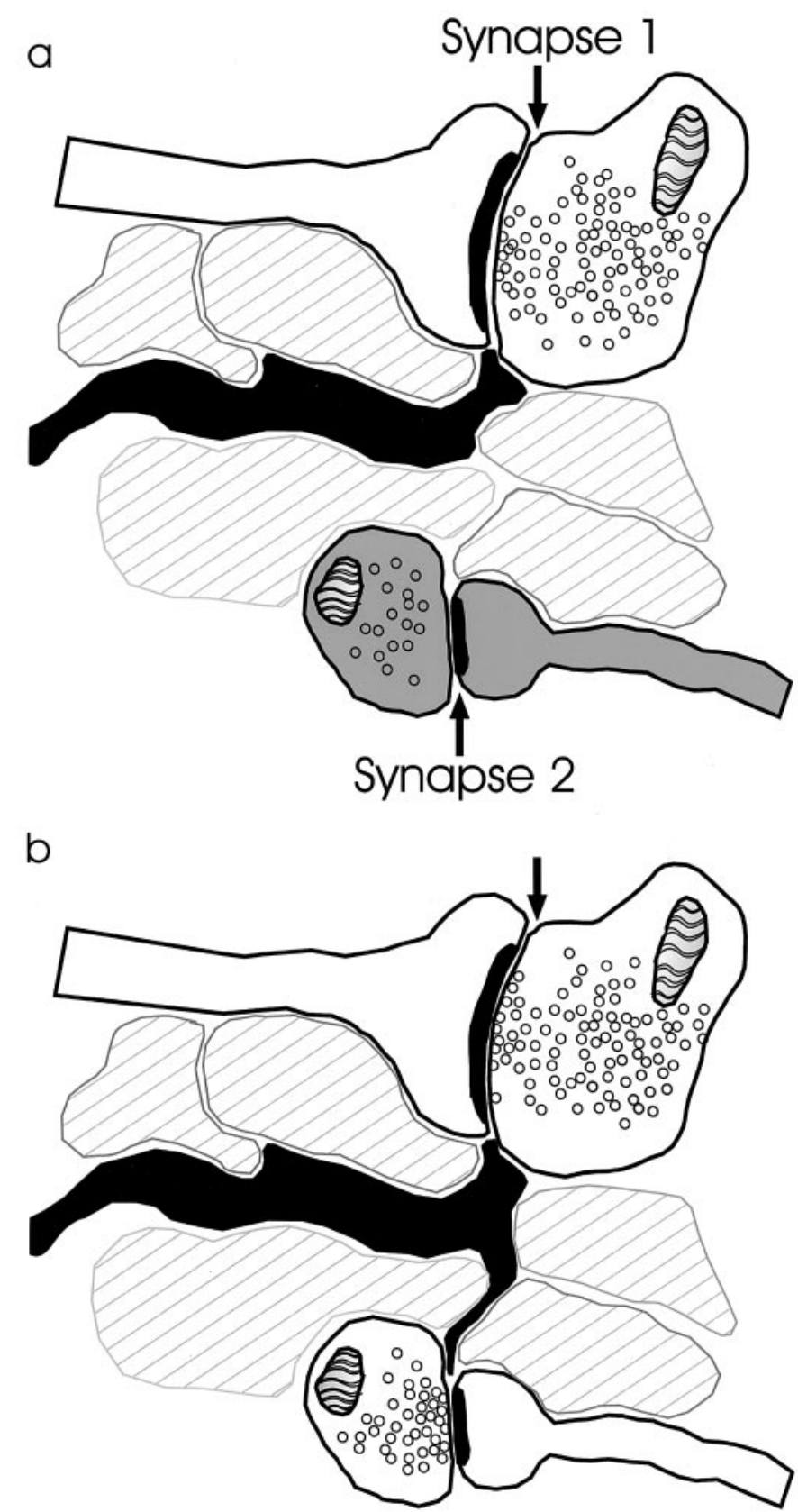

Figure 8. Hypothetical model depicting how the differential distribution of astrocytic processes at hippocampal synapses might reflect synaptic activity. a, A previously releasing synapse (Synapse 1) has an astrocytic process bordering its perimeter where glutamate might otherwise escape from the axon-spine interface. A synapse not releasing glutamate (Synapse 2, dark gray) has no astrocytes bordering its cleft. $b$, The astrocytic process has grown toward Synapse 2 (no longer shaded) as changes in synaptic function have caused it to release sufficient glutamate so that some escapes from its perimeter.

thus the distances between synapses measured on EM sections might be reasonable estimates of the in vivo distances (Lehre and Danbolt, 1998). When the tortuous path through the ECS was measured in $3 \mathrm{D}$, the mean center-to-center distance was $0.65 \mu \mathrm{m}$ in stratum radiatum of area CA1. In Rusakov and Kullmann (1998a,b), a linear distance of $0.436 \mu \mathrm{m}$ was calculated from stereological estimates of synapse density in stratum oriens, and when multiplied by their tortuosity factor of 1.34 , this value becomes $0.584 \mu \mathrm{m}$. Results from the modeling that account for this uniform tortuosity and average linear distance between synapses suggest that the effect of glutamate, even at high-affinity glutamate receptors, will be reduced to $\sim 17 \%$ (Rusakov and Kullmann, 1998a,b). No results have been presented for the larger distances between synapses, although it can be assumed that the effect of glutamate would be further diluted. Given the larger nearest neighboring distances between synapses in stratum radiatum, it is likely that only those receptors located at the edge of some of the PSDs will be exposed to appreciable amounts of glutamate that might have diffused from a neighboring synapse.

Physiological evidence for glutamate spillover between neighboring synapses is controversial. Early evidence for substantial glutamate spillover between hippocampal synapses was obtained in slices maintained at room temperature $\left(\sim 25^{\circ} \mathrm{C}\right)$; however, much less occurs at physiological temperatures $\left(\sim 37^{\circ} \mathrm{C}\right)$ because of an increase in the activity or efficiency of glutamate transporters (Asztely et al., 1997). The surface density of glutamate transporters on hippocampal astrocytic membranes is high $(\sim 10,800$ $\mu \mathrm{m}^{2}$ ) (Lehre and Danbolt, 1998), but the rate of transport is slow (on the order of milliseconds). Thus, the main effect of astrocytic glutamate transporters on the submillisecond time scale will be to bind glutamate, thereby buffering it from the extracellular space (Diamond and Jahr, 1997; Rusakov and Kullmann, 1998a,b). If the astrocytes were the only source of glutamate binding and uptake, one would not expect temperature to have a profound effect on spillover because two-thirds of the neighboring CA1 synapses have no astrocytic processes between them.

One possible explanation is that neuronal glutamate transporters also remove glutamate from the axon-spine interface and ECS between neighboring synapses. Whole-cell recordings from CA1 pyramidal cells have not detected neuronal glutamate transporter currents (Bergles and Jahr, 1998). However, if the neuronal glutamate transporters are located at the synapse (Gundersen et al., 1993; Rothstein et al., 1994; Lehre and Danbolt, 1998), then whole-cell recordings made at the soma would not detect them. GLT1 was previously thought to be strictly an astrocytic glutamate transporter; however, a variant of the GLT1 transporter may also occur on neurons (Torp et al., 1994, 1997; Berger and Hediger, 1998; Chen et al., 1998; Eliasof et al., 1998). Recordings from CA1 pyramidal cells show that glutamate is cleared less quickly during synaptic activation from within the synaptic cleft in the GLT1 (-/-) knockout mice (Tanaka et al., 1997) than in wild-type mice, which may be attributable to the absence of neuronal GLT1 at the axon-spine interface. Neuronal GLT1 might also regulate glutamate along the fine distal axonal and dendritic processes that separate neighboring synapses.

Another possibility is that only the synapses that are releasing substantial amounts of glutamate have astrocytic processes at their perimeters (Fig. 8a). Whole-cell recordings from hippocampal astrocytes show that astrocytic glutamate transporter currents increase in parallel with transiently elevated presynaptic release of neurotransmitter but do not remain elevated during LTP (Diamond et al., 1998; Luscher et al., 1998). Thus, the astrocytic processes are sampling synapses that are reliably releasing glutamate but do not have increased release during LTP. Other research suggests that LTP is partly or fully saturated at $\sim 52 \%$ of hippocampal synapses, so that subsequent experimental manipulations produce little or no more LTP (Petersen et al., 1998). These synapses might account for the $\sim 58 \%$ of hippocampal synapses that have astrocytic processes located at their perimeters. What about the synapses that have no astrocytic processes at 
their perimeters? They might undergo a change in the reliability or amount of glutamate released during synaptic plasticity (Stevens and Wang, 1994; Liu et al., 1999; Ma et al., 1999). As discussed above, astrocytic processes could grow toward a synapse once glutamate levels get high enough for escape to occur from its perimeter (Fig. 8b). If such an astrocytic response occurs quickly, or at low concentrations of extracellular glutamate, then the newly formed astrocytic processes might interrupt spillover and improve input specificity at newly functional synapses.

\section{REFERENCES}

Anderson BJ, Li X, Alcantara AA, Isaacs KR, Black JE, Greenough WT (1994) Glial hypertrophy is associated with synaptogenesis following motor-skill learning, but not with angiogenesis following exercise. Glia 11:73-80.

Asztely F, Erdemli G, Kullmann DM (1997) Extrasynaptic glutamate spillover in the hippocampus: dependence on temperature and the role of active glutamate uptake. Neuron 18:281-293.

Barbour B, Hausser M (1997) Intersynaptic diffusion of neurotransmitter. Trends Neurosci 20:377-384.

Barbour B, Keller BU, Llano I, Marty A (1994) Prolonged presence of glutamate during excitatory synaptic transmission to cerebellar Purkinje cells. Neuron 12:1331-1343.

Berger UV, Hediger MA (1998) Comparative analysis of glutamate transporter expression in rat brain using differential double in situ hybridization. Anat Embryol (Berl) 198:13-30.

Bergles DE, Jahr CE (1997) Synaptic activation of glutamate transporters in hippocampal astrocytes. Neuron 19:1297-1308.

Bergles DE, Jahr CE (1998) Glial contribution to glutamate uptake at Schaffer collateral-commissural synapses in the hippocampus. J Neurosci 18:7709-7716.

Bergles DE, Dzubay JA, Jahr CE (1997) Glutamate transporter currents in bergmann glial cells follow the time course of extrasynaptic glutamate. Proc Natl Acad Sci USA 94:14821-14825.

Chaudhry FA, Lehre KP, van Lookeren C, Ottersen OP, Danbolt NC, Storm-Mathisen J (1995) Glutamate transporters in glial plasma membranes: highly differentiated localizations revealed by quantitative ultrastructural immunocytochemistry. Neuron 15:711-720.

Chen W, Hadley R, Gruber C, Irwin N, Rosenberg PA (1998) Glutamate transporter expression on rat forebrain neurons in culture. Soc Neurosci Abstr 825.5:2066.

Choi DW (1988) Glutamate neurotoxicity and diseases of the nervous system. Neuron 1:623-634.

Cornell-Bell AH, Finkbeiner SM, Cooper MS, Smith SJ (1990) Glutamate induces calcium waves in cultured astrocytes: long-range glial signaling. Science 247:470-473.

Cragg B (1980) Preservation of extracellular space during fixation of the brain for electron microscopy. Tissue Cell 12:63-72.

Diamond JS, Jahr CE (1997) Transporters buffer synaptically released glutamate on a submillisecond time scale. J Neurosci 17:4672-4687.

Diamond JS, Bergles DE, Jahr CE (1998) Glutamate release monitored with astrocyte transporter currents during LTP. Neuron 21:425-433.

Eliasof S, Arriza JL, Leighton BH, Kavanaugh MP, Amara SG (1998) Excitatory amino acid transporters of the salamander retina: identification, localization, and function. J Neurosci 18:698-712.

Engert F, Bonhoeffer T (1997) Synapse specificity of long-term potentiation breaks down at short distances. Nature [Erratum (1997) 388:698] 388:279-284.

Gundersen V, Danbolt NC, Ottersen OP, Storm-Mathisen J (1993) Demonstration of glutamate/aspartate uptake activity in nerve endings by use of antibodies recognizing exogenous D-aspartate. Neuroscience 57:97-111.

Gundersen V, Shupliakov O, Brodin L, Ottersen OP, Storm-Mathisen J (1995) Quantification of excitatory amino acid uptake at intact glutamatergic synapses by immunocytochemistry of exogenous D-aspartate. J Neurosci 15:4417-4428.

Harris KM (1995) How multiple-synapse boutons could preserve input specificity during an interneuronal spread of LTP. Trends Neurosci 18:365-369.

Harris KM, Rosenberg PA (1993) Localization of synapses in rat cortical cultures. Neuroscience 53:495-508.

Harris KM, Stevens JK (1989) Dendritic spines of CA1 pyramidal cells in the rat hippocampus: serial electron microscopy with reference to their biophysical characteristics. J Neurosci 9:2982-2997.

Harris KM, Sultan P (1995) Variation in number, location, and size of synaptic vesicles provides an anatomical basis for the non-uniform probability of release at hippocampal CA1 synapses. J Neuropharmacol 34:1387-1395.

Harris KM, Jensen FE, Tsao B (1992) Three-dimensional structure of dendritic spines and synapses in rat hippocampus (CA1) at postnatal day 15 and adult ages: implications for the maturation of synaptic physiology and long-term potentiation. J Neurosci 12:2685-2705.

Hatten ME (1985) Neuronal regulation of astroglial morphology and proliferation in vitro. J Cell Biol 100:384-396.

Hawrylak N, Chang FL, Greenough WT (1993) Astrocytic and synaptic response to kindling in hippocampal subfield CA1. II. Synaptogenesis and astrocytic process increases to in vivo kindling. Brain Res 603:309-316.

Hessler NA, Shirke AM, Malinow R (1993) The probability of transmitter release at a mammalian central synapse. Nature 366:569-572.

Hillman H, Deutsch K (1978) Area changes in slices of rat brain during preparation for histology or electron microscopy. J Microsc 114:77-84.

Jones TA, Greenough WT (1996) Ultrastructural evidence for increased contact between astrocytes and synapses in rats reared in a complex environment. Neurobiol Learn Mem 65:48-56.

Kullmann DM, Asztely F (1998) Extrasynaptic glutamate spillover in the hippocampus: evidence and implications. Trends Neurosci 21:8-14.

Kullmann DM, Erdemli G, Asztely F (1996) LTP of AMPA and NMDA receptor-mediated signals: evidence for presynaptic expression and extrasynaptic glutamate spill-over. Neuron 17:461-474.

Laming PR, Sykova E, Reichenbach A, Hatton GI, Bauer H (1998) Glial cells: their role in behaviour. Cambridge, UK: Cambridge UP.

Lehre KP, Danbolt NC (1998) The number of glutamate transporter subtype molecules at glutamatergic synapses: chemical and stereological quantification in young adult rat brain. J Neurosci 18:8751-8757.

Linden DJ (1997) Long-term potentiation of glial synaptic currents in cerebellar culture. Neuron 18:983-994.

Linden DJ (1998) Synaptically evoked glutamate transport currents may be used to detect the expression of long-term potentiation in cerebellar culture. J Neurophysiol 79:3151-3156.

Liu G, Choi S, Tsien RW (1999) Variability of neurotransmitter concentration and nonsaturation of postsynaptic AMPA receptors at synapses in hippocampal cultures and slices. Neuron 22:395-409.

Lujan R, Roberts JD, Shigemoto R, Ohishi H, Somogyi P (1997) Differential plasma membrane distribution of metabotropic glutamate receptors mGluR1 alpha, mGluR2 and mGluR5, relative to neurotransmitter release sites. J Chem Neuroanat 13:219-241.

Luscher C, Malenka RC, Nicoll RA (1998) Monitoring glutamate release during LTP with glial transporter currents. Neuron 21:435-441.

Ma L, Zablow L, Kandel ER, Siegelbaum SA (1999) Cyclic AMP induces functional presynaptic boutons in hippocampal CA3- CA1 neuronal cultures. Nat Neurosci 2:24-30.

Malenka RC, Nicoll RA (1997) Silent synapses speak up. Neuron 19:473-476.

Matsutani S, Yamamoto N (1997) Neuronal regulation of astrocyte morphology in vitro is mediated by GABAergic signaling. Glia 20:1-9.

Mennerick S, Benz A, Zorumski CF (1996) Components of glial responses to exogenous and synaptic glutamate in rat hippocampal microcultures. J Neurosci 16:55-64.

Murphy DD, Segal M (1997) Morphological plasticity of dendritic spines in central neurons is mediated by activation of cAMP response element binding protein. Proc Natl Acad Sci USA 94:1482-1487.

Nicholson C, Sykova E (1998) Extracellular space structure revealed by diffusion analysis. Trends Neurosci 21:207-215.

Palay SL, Chan-Palay V (1974) Cerebellar cortex: cytology and organization. New York: Springer.

Parpura V, Basarsky TA, Liu F, Jeftinija K, Jeftinija S, Haydon PG (1994) Glutamate-mediated astrocyte-neuron signalling. Nature 369:744-747.

Peters A, Palay SL, Webster H (1991) The fine structure of the nervous system: the neurons and supporting cells. Philadelphia: W. B. Saunders.

Petersen CC, Malenka RC, Nicoll RA, Hopfield JJ (1998) All-or-none potentiation at CA3-CA1 synapses. Proc Natl Acad Sci USA 95:4732-4737.

Pfrieger FW, Barres BA (1996) New views on synapse-glia interactions. Curr Opin Neurobiol 6:615-621. 
Pfrieger FW, Barres BA (1997) Synaptic efficacy enhanced by glial cells in vitro. Science 277:1684-1687.

Pomeroy SL, Purves D (1988) Neuron/glia relationships observed over intervals of several months in living mice. J Cell Biol 107:1167-1175.

Porter JT, McCarthy KD (1996) Hippocampal astrocytes in situ respond to glutamate released from synaptic terminals. J Neurosci 16:5073-5081.

Porter JT, McCarthy KD (1997) Astrocytic neurotransmitter receptors in situ and in vivo. Prog Neurobiol 51:439-455.

Rosenberg, PA, Aizenman E (1989) Hundred-fold increase in neuronal vulnerability to glutamate toxicity in astrocyte-poor cultures of rat cerebral cortex. Neurosci Lett 103:162-168.

Rosenberg PA, Amin S, Leitner M (1992) Glutamate uptake disguises neurotoxic potency of glutamate agonists in cerebral cortex in dissociated cell cultures. J Neurosci 12:56-61.

Rothstein JD, Martin L, Levey AI, Dykes-Hoberg M, Jin L, Wu D, Nash N, Kuncl RW (1994) Localization of neuronal and glial glutamate transporters. Neuron 13:713-725.

Rothstein JD, Dykes-Hoberg M, Pardo CA, Bristol LA, Jin L, Kuncl RW, Kanai Y, Hediger MA, Wang Y, Schielke JP, Welty DF (1996) Knockout of glutamate transporters reveals a major role for astroglial transport in excitotoxicity and clearance of glutamate. Neuron 16:675-686.

Rusakov DA, Kullmann DM (1998a) Extrasynaptic glutamate diffusion in the hippocampus: ultrastructural constraints, uptake, and receptor activation. J Neurosci 18:3158-3170.

Rusakov DA, Kullmann DM (1998b) Geometric and viscous components of the tortuosity of the extracellular space in the brain. Proc Natl Acad Sci USA 95:8975-8980.

Schousboe A, Westergaard N, Waagepetersen HS, Larsson OM, Bakken IJ, Sonnewald U (1997) Trafficking between glia and neurons of TCA cycle intermediates and related metabolites. Glia 21:99-105.

Schuz A, Palm G (1989) Density of neurons and synapses in the cerebral cortex of the mouse. J Comp Neurol 286:442-455.

Sirevaag AM, Greenough WT (1991) Plasticity of GFAP-immunoreactive astrocyte size and number in visual cortex of rats reared in complex environments. Brain Res 273:278.
Smith SJ (1998) Glia help synapses form and function. Curr Biol 8:R158-R160.

Sonnewald U, Westergaard N, Schousboe A (1997) Glutamate transport and metabolism in astrocytes. Glia 21:56-63.

Sorra KE, Harris KM (1998) Stability in synapse number and size at 2 $\mathrm{hr}$ after long-term potentiation in hippocampal area CA1. J Neurosci 18:658-671.

Spacek J (1985) Three-dimensional analysis of dendritic spines. III. Glial sheath. Anat Embryol (Berl) 171:245-252.

Spacek J, Harris KM (1998) Three-dimensional organization of cell adhesion junctions at synapses and dendritic spines in area CA1 of the rat hippocampus. J Comp Neurol 393:58-68.

Stevens CF, Wang Y (1994) Changes in reliability of synaptic function as a mechanism for plasticity. Nature 371:704-707.

Takahashi M, Billups B, Rossi D, Sarantis M, Hamann M, Attwell D (1997) The role of glutamate transporters in glutamate homeostasis in the brain. J Exp Biol 200:401-409.

Tanaka K, Watase K, Manabe T, Yamada K, Watanabe M, Takahashi K, Iwama H, Nishikawa T, Ichihara N, Kikuchi T, Okuyama S, Kawashima N, Hori S, Takimoto M, Wada K (1997) Epilepsy and exacerbation of brain injury in mice lacking the glutamate transporter GLT-1. Science 276:1699-1702.

Theodosis DT, MacVicar B (1996) Neurone-glia interactions in the hypothalamus and pituitary. Trends Neurosci 19:363-367.

Torp R, Danbolt NC, Babaie E, Bjoras M, Seeberg E, Storm-Mathisen J, Ottersen OP (1994) Differential expression of two glial glutamate transporters in the rat brain: an in situ hybridization study. Eur J Neurosci 6:936-942.

Torp R, Hoover F, Danbolt NC, Storm-Mathisen J, Ottersen OP (1997) Differential distribution of the glutamate transporters GLT1 and rEAAC1 in rat cerebral cortex and thalamus: an in situ hybridization analysis. Anat Embryol (Berl) 195:317-326.

Verkhratsky A, Kettenmann H (1996) Calcium signalling in glial cells. Trends Neurosci 19:346-352.

Vernadakis A (1996) Glia-neuron intercommunications and synaptic plasticity. Prog Neurobiol 49:185-214. 